\title{
Highly oriented and crystalline films of a phenyl- substituted polythiophene prepared by epitaxy : structural model and influence of molecular weight
}

\author{
Amer Hamidi-Sakr ${ }^{1}$, Daniel Schiefer ${ }^{2}$, Sangeetha Covindarassou ${ }^{1}$, Laure \\ Biniek $^{1}$, Michael Sommer ${ }^{2,3,4}$, Martin Brinkmann ${ }^{1}$
}

(1) Institut Charles Sadron, CNRS - Université de Strasbourg, 23 rue du loess, 67034 Strasbourg, France

(2) : Institut für Makromolekulare Chemie, Universität Freiburg, Stefan-MeierStraße 31, 79104 Freiburg, Germany

(3) Freiburg Material Research Center, Universität Freiburg, Stefan-MeierStraße 21, 79104 Freiburg, Germany

(4) Freiburg Institute for Interactive Materials and Bioinspired Technologies, Georges-Köhler-Allee 105, 79110 Freiburg, Germany 


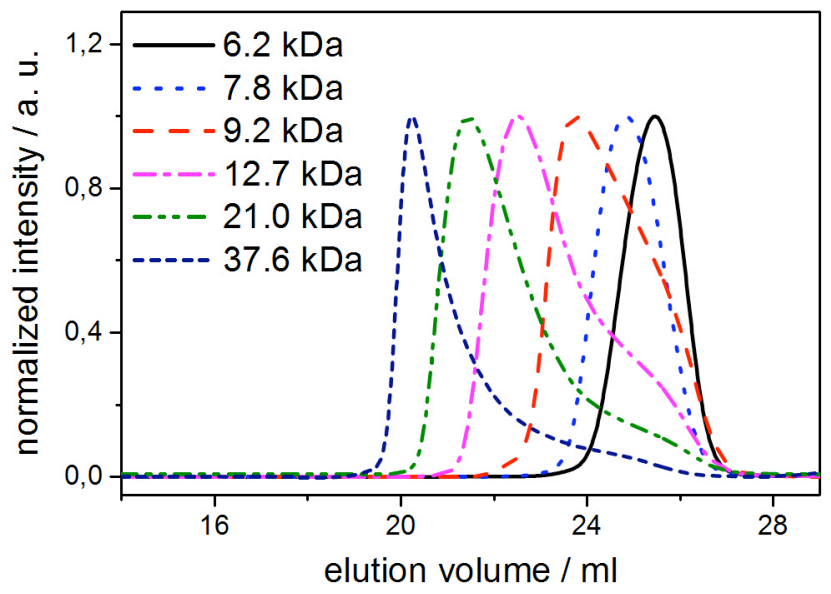

Figure S1. SEC elution profiles of the different PDOPT samples in THF.
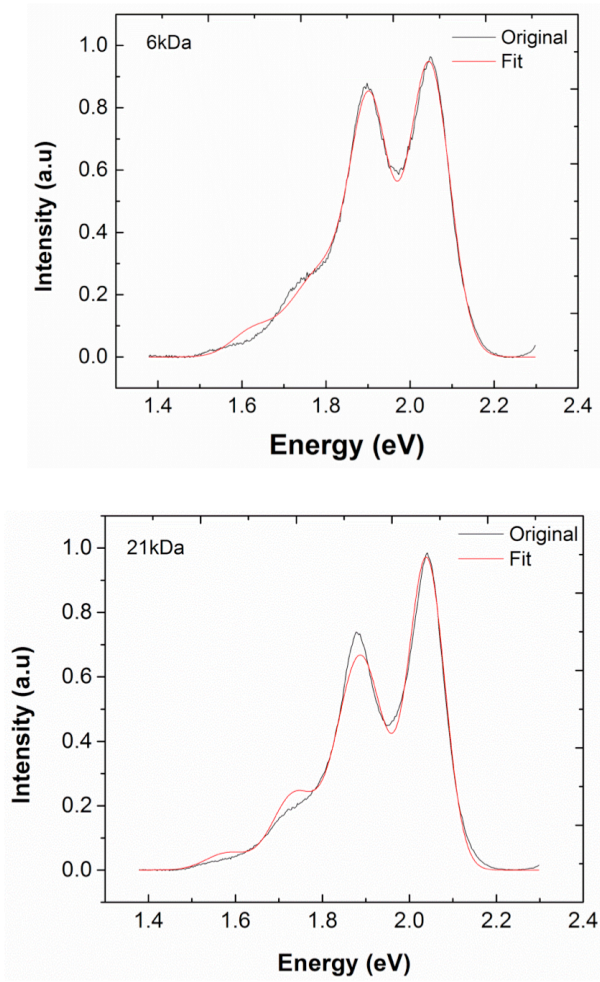

Figure S2. Photoluminescence spectra of oriented PDOPT films prepared by slow-DEC on naphthalene fro two different molecular weights $\left(M_{\mathrm{n}}\right)$. The figures show the fit of the PL spectra using a simple Frank-Condon progression (in red). 


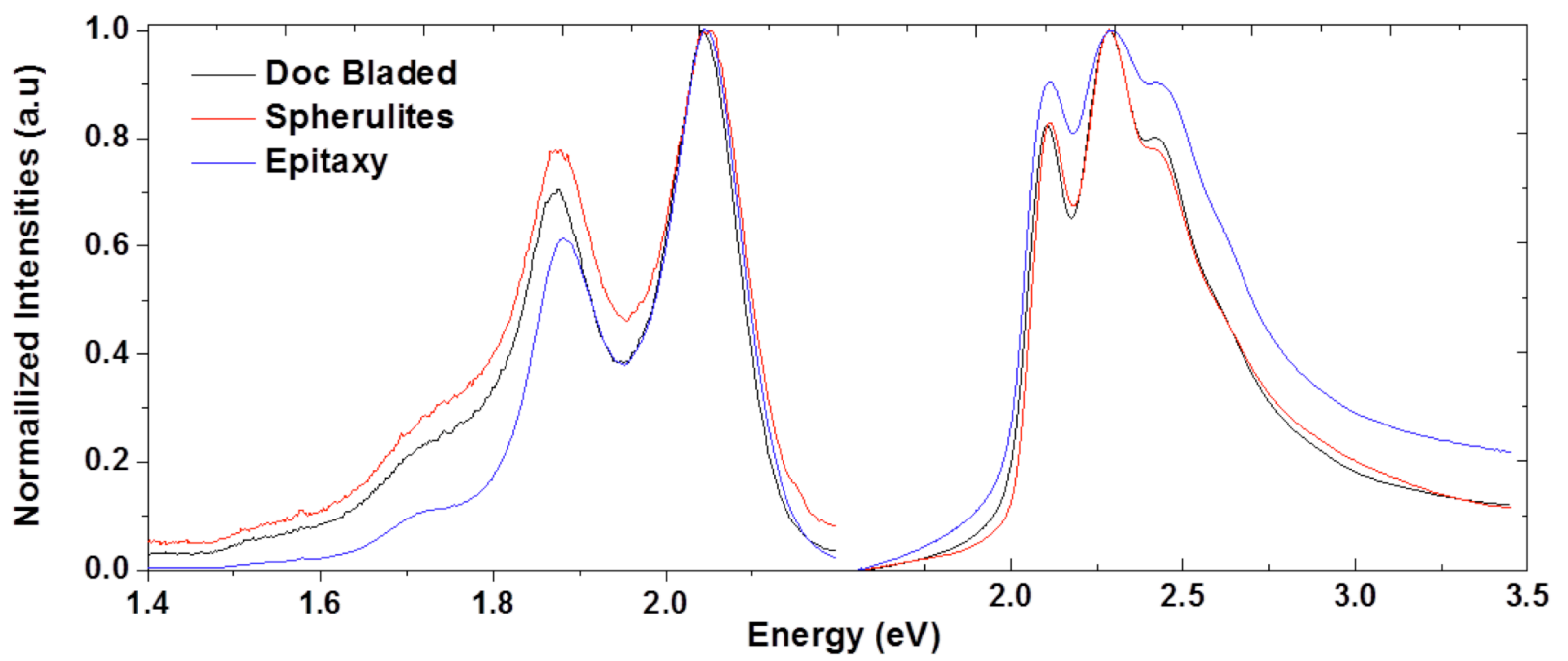

Figure S3. Comparison of the photoluminescence and UV-vis spectra in PDOPT films prepared by doctor blading, isothermal crystallization at $100^{\circ} \mathrm{C}$ and by slow directional epitaxial crystallization on naphthalene. The PL spectra are normalized with respect to the 0-0 vibronic component whereas the absorption spectra are normalized with respect to the $0-1$ component.
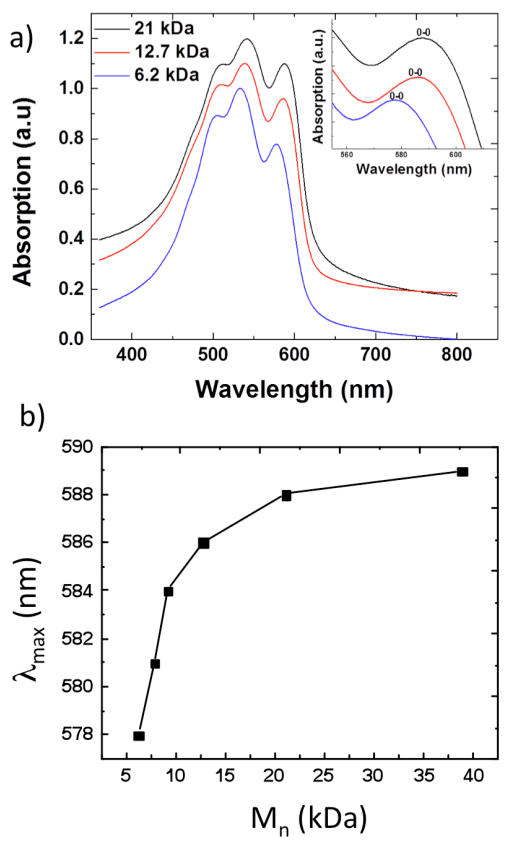

Figure S4. Evolution of optical absorption spectrum of PDOPT films oriented by epitaxy on naphthalene as a function of $M_{\mathrm{n}}$ (incident polarization parallel to the chain direction of PDOPT). The inset shows the shift of the 0-0 transition in the UV-vis absorption spectrum with $\mathrm{M}_{\mathrm{n}}$. b) Evolution of $\lambda_{\max }$ as a function of $M_{\mathrm{n}}$. 
Figure S4 features the evolution of the UV-vis absorption spectrum and $\lambda_{\max }$ versus molecular weight of PDOPT. Regarding, the UV-vis absorption, one observes a $\sim 10 \mathrm{~nm}$ red shift of the 0-0 peak position for the higher $M_{n}$ of $21 \mathrm{kDa}$. The position of the $0-0$ component in epitaxied P3HT films showed a similar red-shift with increasing $M_{n} \cdot{ }^{31}$ From the evolution of lamellar period $L_{p}$ verus $M_{n}$, this trend is possibly related to the impact of reduced crystal size along the chain direction on the position of the $0-0$ peak position. Higher $M_{n}$ PDOPT show longer planarized chain segments in the crystalline domains, hence a larger conjugation length which should translate to a red-shift of the 00 peak.

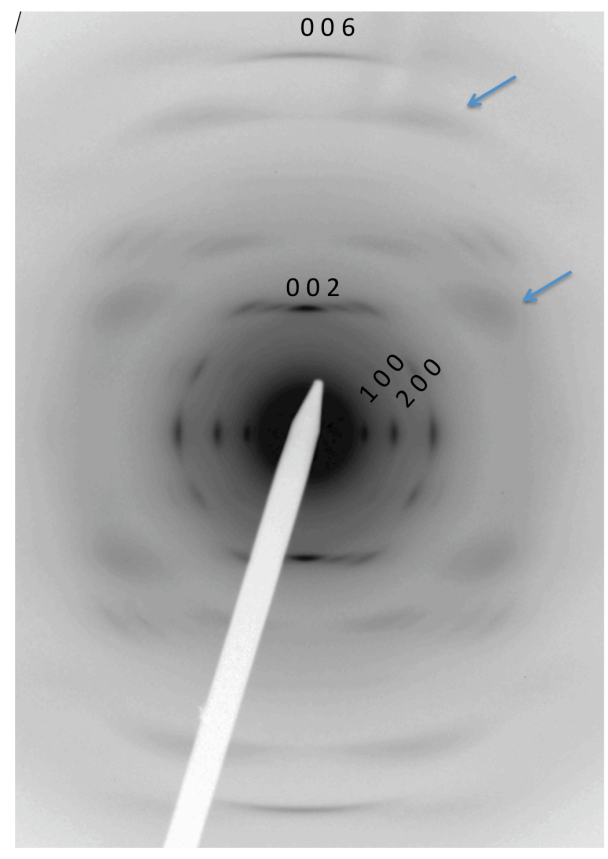

Figure S5. Electron diffraction pattern of a highly crystalline and oriented P3HT film obtained by high-temperature rubbing at $180^{\circ} \mathrm{C}$. The two arrows point at fuzzy reflections indicative of structural disorder in the packing of $n$-hexyl side chains in form I P3HT. 